\title{
Dynamique D’occupation Du Sol Et Diversité Floristique De La Forêt Classée De La Palé (Côte d'Ivoire)
}

\author{
Pagadjovongo Adama Silue, Assistant \\ Dramane Soro, Maître-Assistant \\ Université Péléforo Gbon Coulibaly, Unité de Formation et de Recherches \\ des Sciences Biologiques, Korhogo / Côte d'Ivoire \\ Konan Edouard Kouassi, Maître de Conférences \\ Dodiomon Soro, Professeur Titulaire
}

Université Félix Houphouët-Boigny, Unité de Formation et de Recherches de Biosciences, Abidjan / Côte d'Ivoire

Doi:10.19044/esj.2021.v17n43p1

Submitted: 24 July 2021

Accepted: 14 October 2021

Published: 31 December 2021
Copyright 2021 Author(s)

Under Creative Commons BY-NC-ND 4.0 OPEN ACCESS

Cite As:

Adama Silue P., Soro D., Kouassi E.K.,\& Soro D.,(2021). Dynamique D’occupation Du Sol Et Diversité Floristique De La Forêt Classée De La Palé (Côte d'Ivoire) European Scientific Journal, ESJ, 17(43), 1.

https://doi.org/10.19044/esj.2021.v17n43p1

\section{Résumé}

Les forêts classées du Nord de la Côte d'Ivoire connaissent un phénomène d'anthropisation accrue, notamment à la faveur de la crise sociopolitique de 2002 à 2011. A cela, s'ajoute l'effet des changements climatiques qui ont des conséquences sur la qualité de la biodiversité. Cette étude recherche, à travers l'analyse de paramètres floristiques et de la dynamique d'occupation du sol, des informations scientifiques fiables devant guider les orientations d'aménagement d'une forêt classée située dans la Région de la Bagoué, au Nord-Ouest du pays. L'étude de la dynamique de la couverture végétale de la Forêt classée est réalisée à travers une analyse diachronique des images satellitaires Landsat ETM de janvier 2002 et ETM + de mars 2016. L'appréciation des paramètres floristiques est basée sur l'interprétation des données d'inventaire floristique. Les résultats cartographiques montrent une régression des formations boisées passant de $81,75 \%$ en 2002 à $71,19 \%$ en 2016, soit un taux de régression annuel de 0,7 $\%$. Concernant l'étude floristique, au total 281 espèces végétales, réparties entre 210 genres et rangées dans 71 familles, ont été inventoriées dans la forêt 
classée. Par cette flore, 18 espèces ont présenté un statut particulier, preuve de la valeur de conservation de cette formation végétale. Ce résultat pourrait servir pour la mise en place d’une forêt classée dans la Région de la Bagoué en Côte d'Ivoire.

Mots clés: Biodiversité, Espèces À Statut Particulier, Images Satellitaires, Couverture Végétale, Région De La Bagoué

\title{
Land-Use Dynamics And Floristic Diversity Of Palé Classified Forest In Côte d'Ivoire
}

\section{Pagadjovongo Adama Silue, Assistant} Dramane Soro, Maître-Assistant

Université Péléforo Gbon Coulibaly, Unité de Formation et de Recherches des Sciences Biologiques, Korhogo / Côte d'Ivoire

Konan Edouard Kouassi, Maître de Conférences

Dodiomon Soro, Professeur Titulaire

Université Félix Houphouët-Boigny, Unité de Formation et de Recherches de Biosciences, Abidjan / Côte d'Ivoire

\begin{abstract}
The classified forests of the north of Ivory Coast are experiencing an increased anthropization phenomenon, in particular thanks to the sociopolitical crisis from 2002 to 2011. In addition to this is the effect of climate change that has consequences on the quality of biodiversity. This paper focuses on the analysis of floristic parameters, the dynamics of land use, and reliable scientific information to guide the development orientations of a classified forest located in the Bagoué Region in the North-West. The study of the dynamics of the vegetation cover of the classified forest was carried out through a diachronic analysis of the Landsat ETM images of January 2002 and ETM + of March 2016. The appreciation of the floristic parameters is based on the interpretation of the floristic inventory data. The cartographic results show a regression of woodlands from $81.75 \%$ in 2002 to $71.19 \%$ in 2016 , i.e., an annual rate of decline of $0.7 \%$. Concerning the floristic study, a total of 281 plant species, divided between 210 genera and arranged in 71 families, were inventoried in the classified forest. Through this flora, 18 species presented a special status, which is a proof of the conservation value of this plant formation. This result could be used for the establishment of a classified forest in Bagoué Region of Côte d’Ivoire.
\end{abstract}


Keywords: Biodiversity, Special-Status Species, Satellite Images, Plant Cover, Bagoué Region

\section{Introduction}

En Afrique occidentale, de nombreux auteurs (Hien et al., 2002 ; Grégroire \& Simonetti, 2010) soutiennent que les formes dominantes de perturbations de la structure et de la physionomie de la végétation au sein des aires protégées sont les feux de végétation, le pâturage, l'agriculture itinérante sur brulis et l’irrégularité de la pluviométrie.

En Côte d'Ivoire, cette régression du couvert forestier s'explique par l'option d'une économie basée sur la valorisation des ressources naturelles et l'agriculture dont les prémisses ont été posées à l'ère coloniale et qui s'est perpétuée après l'accession du pays à l'indépendance en 1960 (Léonard \& Ibo, 1994 ; Balac, 2000). Face à cette forte déforestation les pouvoirs publics ont érigé certaines zones en forêts classées dans le but de freiner la dynamique de dégradation et d'exploitations abusives des forêts et de la biodiversité. En effet, les forêts classées, comme c'est le cas des réserves naturelles et les parcs nationaux, sont censées représenter des zones de conservation privilégiées de la flore et de la faune (Tankoano, 2012). Malheureusement, nombreuses sont les forêts classées qui ont subi et subissent encore une forte pression anthropique (exploitation agricoles, infrastructures socioéconomiques, activité agricole, etc.). A ces contraintes anthropiques, il faut ajouter les effets des changements climatiques globaux (les sécheresses récurrentes, les déficits pluviométriques, le réchauffement).

C'est le cas des forêts classées situées au Nord du pays, qui ont été soustraites du contrôle de l'Etat à la faveur de la crise politico-militaire de 2002 à 2010. Devant une problématique aussi complexe, l'observation des mutations du paysage forestier au cours du temps s'impose afin d'estimer la nature de l'évolution des processus naturels et anthropiques pour une gestion durable des ressources naturelles (Inoussa et al., 2011). Ainsi, après la fin de la crise en 2011, il serait bon de faire un état des lieux afin d'actualiser les données tant sur les surfaces forestières restantes que sur la structure de la végétation et sa composition floristique. Ces informations sont en effet, indispensables pour susciter un intérêt auprès des décideurs et bailleurs de fonds quant à la réhabilitation des espaces dégradés. C'est pour répondre à cette préoccupation qu'a été initiée la présente étude sur la forêt classée de la Palé, dans le Nord-Ouest de la Côte d'Ivoire. L'hypothèse de cette étude est que les infiltrations humaines liées à l'absence de l'autorité de l'Etat et combinées aux variabilités climatiques ont produits des changements sur le couvert végétal dans la forêt classée de la Palé.

L’objectif général est donc de faire l'état des lieux sur la végétation et la flore, en vue de fournir des données scientifiques utiles à l'aménagement de 
cette forêt classée. De façon spécifique, il s’agit d'abord de cartographier et d'analyser la dynamique d'occupation de sol de la forêt classée de la Palé en 2002 et en 2016, ces deux dates correspondent, respectivement, au début de la crise politico-militaire et à la reprise en main de la forêt classée par l'Etat ; de caractériser la flore générale de la forêt classée. Ces informations sont nécessaires aux orientations d'aménagement de gestion durable de cette aire protégée menacée par la pression anthropique.

\section{Materiel Et Methodes}

\section{Description de la zone d'étude}

Incluse dans une vaste zone agropastorale, la forêt classée de la Palé couvre une superficie de 25040 ha. Elle se localise dans le centre de la Région de la Bagoué entre $9^{\circ} 50^{\prime}$ et $9^{\circ} 35^{\prime}$ de latitude Nord et entre $6^{\circ} 45^{\prime}$ et $4^{\circ} 35^{\prime}$ de longitude Ouest (Figure 1). La zone est caractérisée par un climat de type soudanien avec une longue saison sèche de novembre à mai et une courte saison des pluies de juin à octobre. La pluviométrie annuelle oscille entre 1000 et $1400 \mathrm{~mm}$ en moyenne. La végétation est celle des secteurs subsoudanais et soudanais telle que décrite par Kouamé et al. (2010). Les températures moyennes mensuelles sont comprises entre $25^{\circ} \mathrm{C}$ et $31^{\circ} \mathrm{C}$. Le relief est caractérisé par son plan horizontal fait de plaines et de plateaux dont la monotonie est rompue, par endroits, par l'apparition de chaînes de collines ou de dômes rocheux qui varient entre 400 et $600 \mathrm{~m}$ d'altitude. Au niveau pédologique, Les sols comprennent, les lithosols (4 p.c.), les vertisols (39 p.c.) et les sols ferrugineux (57 p.c.) selon Beaudou et Sayol (1980). Le potentiel hydrographique de la forêt classée repose essentiellement sur la rivière Palé, qui est un affluent du fleuve Bagoué, et d'autres petites rivières et marigots temporaires.

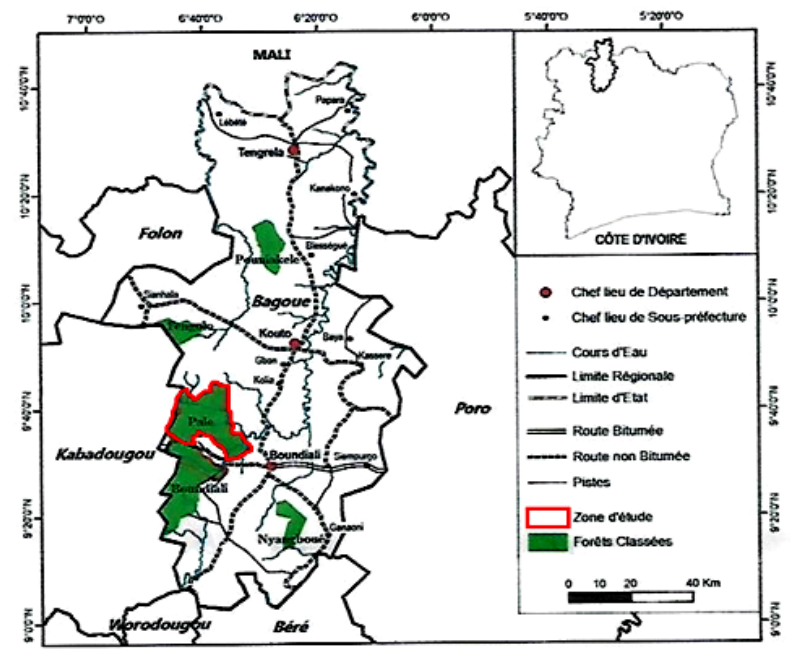

Figure 1. Localisation de la zone d'étude 


\section{Collecte des données \\ Données satellitaires}

L’étude de la dynamique spatiale de la végétation de la forêt classée de la Palé est basée sur l'interprétation d'images satellitaires par une analyse diachronique des images de deux périodes différentes. Les images satellitaires utilisées sont celles de Landsat ETM du 27 janvier 2002 scène 198-53 et Landsat ETM+ du 30 mars scène 198-53. L'image de 2002 a été choisie afin de déterminer la situation de l'occupation du sol peu avant le déclenchement de la crise politico-militaire en 2002. L’image de 2016 correspond à la période de reprise en main de la forêt classée de la Palé par l'administration forestière (SODEFOR). Un GPS (Global Positioning System) a été utilisé pour localiser les points d'échantillonnage pour la reconnaissance et le contrôle de terrain.

\section{Inventaires floristiques}

Une combinaison de méthodes d’échantillonnage a été mise en œuvre pour l'inventaire de la flore de la forêt classée de la Palé. Il s’agit de relevés de surface et de relevés itinérants. Le relevé de surface a été réalisé dans 10 parcelles de 1 ha (100 m x $100 \mathrm{~m})$, subdivisées chacune en des placettes de $100 \mathrm{~m}^{2}$ (10 m x $10 \mathrm{~m}$ ). Cette méthode d'inventaire avec des placettes de 100 $\mathrm{m}^{2}$ comme unité d’échantillonnage a été déjà utilisée par de nombreux auteurs dans des études floristiques (Kouassi, 2007 ; Tiébré et al., 2016). 10 placettes ont été choisies, au hasard, par tirage au sort sans remise. Dans chacune de ces placettes, la présence des végétaux supérieures a été noté, sans tenir compte de leur abondance, ni de leur taille, ni de leur stade phénologique. Le positionnement des parcelles d'inventaires s'est fait à partir des points choisis, arbitrairement, sur la carte de la forêt classée et reparties de façon homogène sur la superficie de ladite forêt. Les inventaires itinérants ont été réalisés entre deux parcelles, le long des pistes et des cours d'eaux, etc. Cette méthode a permis de récolter de nouveaux spécimens de plantes. La liste floristique finale de la forêt classée a été établie avec les espèces issues des relevés itinérants et de surface.

\section{Analyse et traitement des données}

La cartographie a été réalisée suivant trois étapes : le prétraitement des images après leur acquisition, la classification des types d'occupation de sol, la validation des types d'occupation de sol et la réalisation des cartes d’occupation de sol. Le prétraitement d’image a débuté par la correction radiométrique puisque la correction géométrique n’a pas été nécessaire, les images étant déjà géoréférenciées. La correction radiométrique a consisté à normaliser l'image selon les propriétés du capteur Landsat 7 ETM+, de convertir les valeurs numériques des images en valeurs de réflectance selon les paramètres du capteur Landsat 7 ETM+ et de minimiser l'impact des 
conditions no-surfaciques sur les valeurs de l'image de 2002. L'image obtenue étant en niveau gris, il a fallu utiliser la composition colorée pour produire une image couleur à partir de la combinaison de trois bandes spectrales, relatives aux trois couleurs primaires, rouge, vert et bleu (Enonzan, 2010). Les compositions colorées utilisées pour mieux discriminer les types de végétations sont ETM+ 5-4-3. Elles ont permis de sélectionner les sites à visiter, l'orientation sur le terrain, les parcelles d'entrainement pour la réalisation des classifications et les parcelles de contrôle pour l'évaluation des classifications.

Une classification a été effectuée par la méthode des K-Means (classification par les Nuées Dynamiques) pour regrouper les classes d'occupation du sol présentant les mêmes valeurs radiométriques (Bonn \& Rochon, 1993). Elle aboutit à la discrimination d'un nombre de classes élevées qui offre la possibilité de fusionner ultérieurement suivant différents thèmes (forêts, savanes, cultures etc.). En fin, des sites préalablement identifiés sur les images de 2002 et de 2016 ont été visités à partir de leurs coordonnées géographiques. Au total 14 points ont été visités. De façon globale, il s’agit des îlots de forêts, des savanes, des espaces cultivés et d'affleurement granitiques. La caractérisation de la flore a été faite d'abord à travers la détermination du nombre d'espèces, de genres, de familles et des types morphologiques (arbres ou arbuste, liane et herbe). Ces paramètres, ont permis d'avoir une idée globale de la richesse floristique, de la composition floristique et des espèces à statut particulier de la forêt classée. La richesse floristique est définie comme étant le nombre d'espèces recensées sur un territoire donné (Aké-Assi, 1984). La composition floristique renvoie aux caractéristiques de la flore étudiée. Il s'agit notamment de préciser : les familles, les genres, les types biologiques, les affinités chorologiques, et les spécialisations. Les travaux de Aké-Assi $(2001,2002)$ ont servi de base à l'établissement de ces listes. La valeur de la forêt classée pour la conservation de la biodiversité a été analysée à travers la détermination des espèces dites à statut particulier. Il s'agit d'identifier les espèces endémiques ivoiriennes (GCi), les endémiques des forêts de la Haute Guinée (HG), ou du bloc forestier ouest-africain (GCW), sur la base des listes d'espèces préétablies par Aké-Assi (1984 ; 2001 ; 2002), Holmgren et al. (2004). A ces espèces endémiques, a été ajoutée les listes des espèces rares ou menacées d'extinction de la flore ivoirienne selon l'UICN (2015) et des espèces à valeur commerciale.

\section{Resultats}

\section{Vérification de la classification dirigée}

La discrimination entre les classes thématiques (unités d'occupation des sols), obtenue à partir des matrices de confusions (Tableaux 1 et 2), donne pour chacune des classifications des précisions globales variant de 93,89\% à 
95,23\% et des valeurs du coefficient Kappa élevés avec 0,92 et 0,93 respectivement pour les images 2016 et 2002. Les valeurs en gras dans la diagonale des tableaux correspondent pour chaque type d'occupation, aux taux de pixels bien classés, c'est-à-dire les taux de pixels d'une classe effectivement affectée à celle-ci par la méthode de classification utilisée. On remarque que toutes les classes sont bien discriminées tant pour l'image de 2002 (précision $\geq 91,67 \%$ ) que pour l'image de 2016 (précision $\geq 89,33 \%$ ). Cependant, on note quelques confusions entre les classes dont les plus élevées existent, en 2016, entre forêt claire et savane arborée $(10,77)$ et entre complexe forêt dense sèche/forêt galerie et forêt claire (5,88\%). En ce qui concerne la classification de l'image de 2001 (Tableau 1), les confusions les plus importantes ont été enregistrées entre affleurements granitiques et savane arborée.

Tableau 1. Matrice de Confusion de l’image Landsat ETM 2002 de la forêt classée de la Palé

\begin{tabular}{|c|c|c|c|c|}
\hline & $\begin{array}{c}\text { Forêt dense sèche/forêt } \\
\text { galerie }\end{array}$ & $\begin{array}{c}\text { Forêt } \\
\text { claire }\end{array}$ & $\begin{array}{c}\text { Savane } \\
\text { arborée }\end{array}$ & $\begin{array}{c}\text { Affleurements } \\
\text { granitiques }\end{array}$ \\
\hline $\begin{array}{c}\text { Forêt dense } \\
\text { sèche/forêt galerie }\end{array}$ & 100 & 3,16 & 0 & 0 \\
\hline Forêt claire & 0 & 94,74 & 2,78 & 0 \\
\hline Savane arborée & 0 & 2,11 & 91,67 & 1,3 \\
\hline $\begin{array}{c}\text { Affleurements } \\
\text { granitiques }\end{array}$ & 0 & 0 & 5.56 & 98,7 \\
\hline Totaux & 100 & 100 & 100 & 100 \\
\hline
\end{tabular}

Précision globale $=93,89$ p.c. ; Coefficient Kappa $=$ 92,09 p.c.

Tableau 2. Matrice de Confusion de l'image Landsat ETM+ 2016 de la forêt classée de la Palé

\begin{tabular}{|c|c|c|c|c|c|}
\hline & $\begin{array}{c}\text { Affleurements } \\
\text { granitiques }\end{array}$ & $\begin{array}{c}\text { Savane } \\
\text { arborée }\end{array}$ & $\begin{array}{c}\text { Zone de } \\
\text { cultures }\end{array}$ & $\begin{array}{c}\text { Forêt dense } \\
\text { sèche/Forêt } \\
\text { galerie }\end{array}$ & $\begin{array}{c}\text { Forêt } \\
\text { claire }\end{array}$ \\
\hline $\begin{array}{c}\text { Affleurements } \\
\text { granitiques }\end{array}$ & 94,83 & 0 & 0 & 0 & 0 \\
\hline Savane arborée & 3,69 & 100 & 0,45 & 1,47 & 10,77 \\
\hline Champs & 0 & 0 & 99,55 & 0 & 0 \\
\hline $\begin{array}{c}\text { Forêt dense } \\
\text { sèche/Forêt galerie }\end{array}$ & 1,48 & 0 & 0 & 92,65 & 0 \\
\hline Forêt claire & 0 & 0 & 0 & 5,88 & 89,23 \\
\hline Totaux & 100 & 100 & 100 & 100 & 100 \\
\hline
\end{tabular}

Précision globale $=95,23$ p.c. ; Coefficient Kappa $=93,43$ p.c.

Etat d'occupation du sol en 2002 et 2016

La classification dirigée des images satellitaires a permis de produire la carte de l'occupation de sol pour 2002 (Figure 2a) et pour 2016 (Figure 2b). Sur la carte 2002, la végétation de la forêt classée de la Palé est dominée par la forêt claire avec 7935,18 ha, soit 31,69\% et le complexe forêt dense 
sèche/forêt galerie avec 6878,49 ha, soit $27,47 \%$. Les affleurements granitiques sont la classe d'occupation la moins représentée avec 4552,27 ha, soit $18,18 \%$. On note une absence des zones de cultures sur la carte d'occupation du sol de la forêt.

Sur la carte d'occupation de sol de 2016, la forêt claire, avec 8158,03 ha, soit 32,58\%, domine toujours la végétation de la forêt. Suivent les affleurements granitiques avec 6422,76 ha, soit $25,65 \%$ et les savanes arborées avec 4950,41 ha, soit 19,77\% et les forêts denses sèches/forêts galeries avec 4702,51 ha, soit 18,78\%. Les zones de cultures apparues en 2016, constituent la classe d'occupation la moins représentée avec 806,29 ha, soit 3,22\% et sont localisées au nord de la forêt classée.

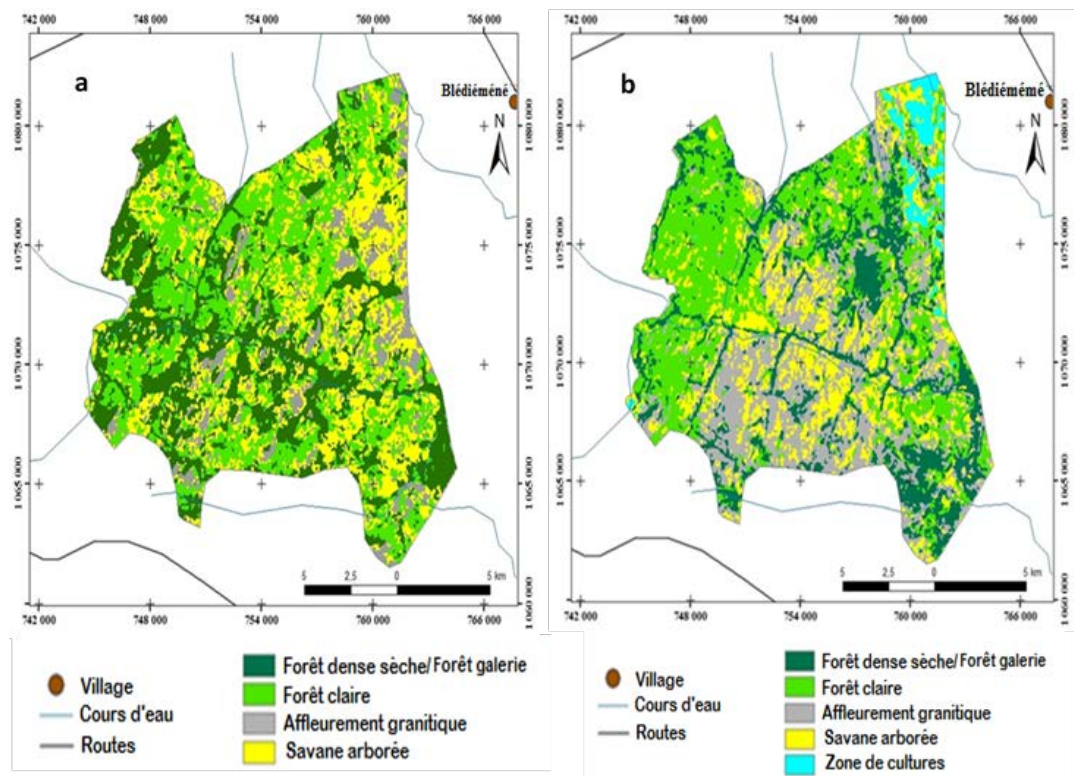

Figure 2. Carte des types d'occupation de sol de la forêt classées de la Palé à partir de l'image Landsat ETM de 2002 (a) et de l'image Landsat ETM+ de 2016 (b)

\section{Dynamique des classes d'occupation du sol entre 2002 et 2016}

Les résultats cartographiques et statistiques obtenus permettent de mettre en exergue la dynamique des classes d'occupation du sol entre 2002 et 2016 (Figure 3). On note d'une part une régression du complexe forêt dense sèche/forêt galerie qui passe de 6878,49 ha à 4702,51 ha, soit une perte de 145,06 ha/an. On remarque que la savane arborée a également diminué en superficie, passant de 5674,06 ha à 4950,41 ha, soit un taux de 2,93\% de la surface de la forêt classée. D'autre part, on observe une augmentation des superficies occupées par la forêt claire entre 2002 et 2016. La forêt claire a gagné 222,85 ha entre 2002 et 2016, soit un taux d'évolution de 0,91\% sur la période d'étude. On constate aussi une augmentation de la classe des affleurements granitiques de 1870,49 ha, soit une augmentation de 124,70 
ha/an et une apparition de zones de cultures de 3,22\% en 2016. On constate dès lors des changements dans la structure de l'occupation du sol dans la forêt classée de la Palé durant la période de 2002 à 2016. L’analyse du vecteur de changement montre que $15,03 \%$ de la forêt a connu une évolution positive (augmentation de la radiométrie) et, 17,56\%, une évolution négative (baisse de la radiométrie). On observe, par ailleurs, que $67,41 \%$ de la forêt sont restés stable de 2002 à 2016 (Figure 4).

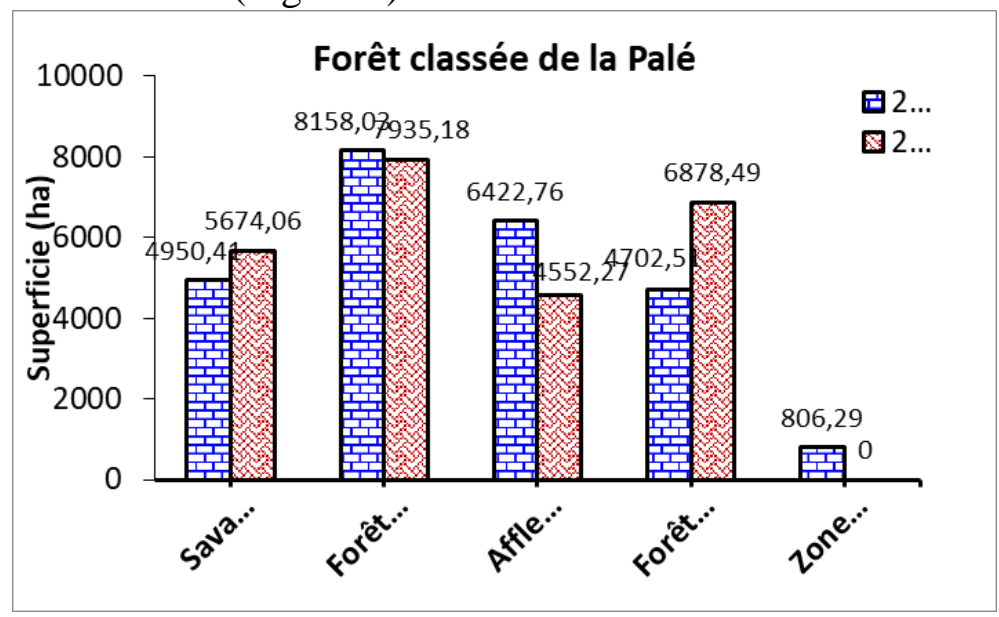

Figure 3. Dynamiques d’occupation du sol dans la forêt classée de la Palé en 2002 et en 2016

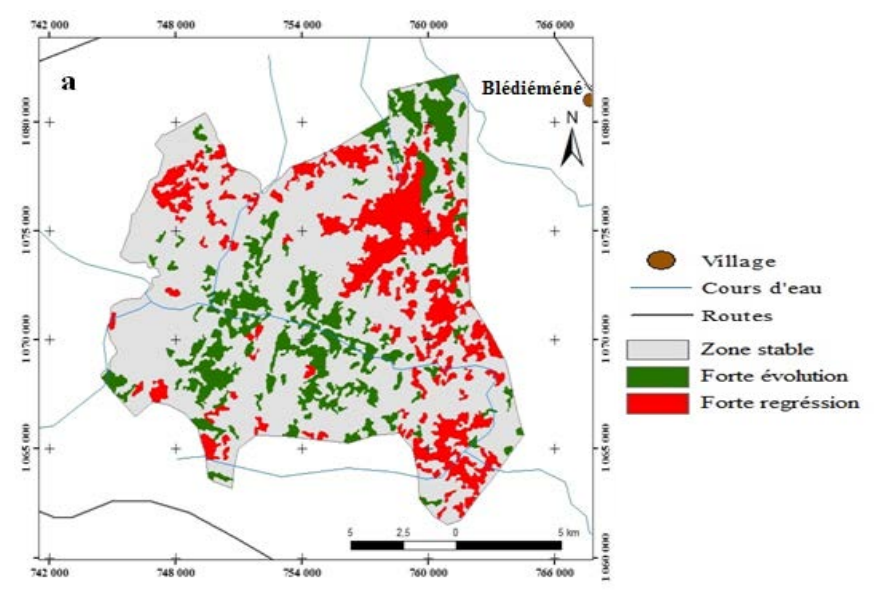

Figure 4. Dynamique de la végétation des forêts classées de la Palé entre 2002 et 2016

\section{Caractérisation de la flore}

\section{Richesse et composition floristiques}

L’inventaire itinérant et de relevé de surface, a permis de dénombrer 281 espèces reparties entre 210 genres rangés dans 71 familles. Les familles dominantes, par ordre décroissant du nombre d'espèces, sont les Fabaceae (59 
espèces), les Poaceae (17 espèces), les Rubiaceae (14 espèces), les Malvaceae (12 espèces), et les Phyllanthaceae (11 espèces) (Figure 5).

L'analyse de la richesse floristique des Angiospermes dans la forêt classée met en évidence la prépondérance des Dicotylédones avec 223 espèces (79,36 p.c.) réparties entre 163 genres regroupés au sein de 55 familles. Les Monocotylédones regroupent 56 espèces (19,93 p.c.) réparties entre 45 genres que partagent 14 familles. Les Ptéridophytes sont faiblement représentées par deux espèces ( 0,70 p.c.), deux genres et deux familles (Tableau 3).

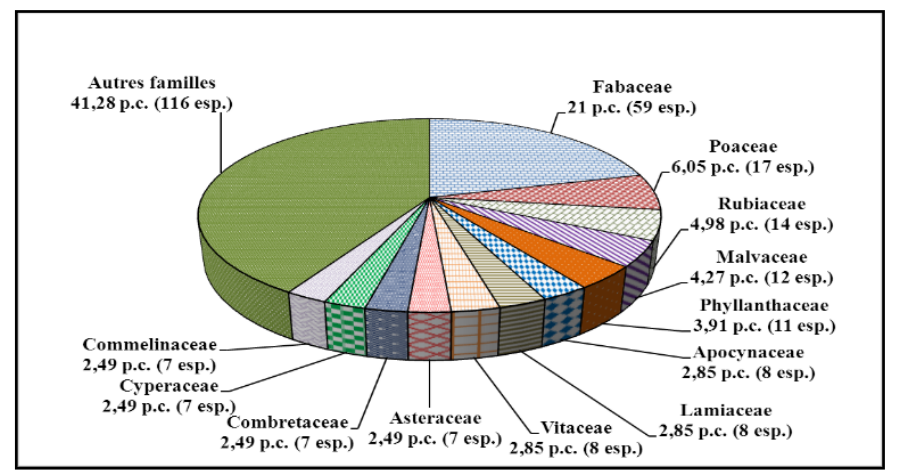

Figure 4. Spectre des familles dominantes de la flore de la forêt classée de la Palé

Tableau 3. Répartition des espèces inventoriées dans les deux forêts classées en taxons supérieurs

\begin{tabular}{|c|c|c|c|c|}
\hline & Dicotylédones & Monocotylédones & Ptéridophytes & Total \\
\hline Nombre d'espèces & 223 & 56 & 2 & 281 \\
\hline Taux (p.c.) & 79,36 & 19,93 & 0,71 & 100 \\
\hline Nombre de genres & 163 & 45 & 2 & 210 \\
\hline Taux (p.c.) & 77,62 & 21,43 & 0,95 & 100 \\
\hline Nombre de familles & 55 & 14 & 2 & 71 \\
\hline Taux (p.c.) & 77,47 & 19,72 & 2,82 & 100 \\
\hline
\end{tabular}

\section{Diversité des types biologiques}

Les spectres biologiques des espèces inventoriées (Figure 5) mettent en évidence une nette dominance des Phanérophytes avec 197 espèces, soit 70,10 p.c. du total des espèces. Les Chaméphytes, les Epiphytes et les Hydrophytes sont les types bioloquiques les moins représentés avec, respectivement, 4 espèces (42 p.c.), 5 espèces $(1,77$ p.c.) et 1 espèce $(0,35$ p.c.). Parmi les Phanérophytes, 4 espèces, soit 1,43 p.c. de l'effectif total, appartiennent à la strate haute (hauteur supérieure à $32 \mathrm{~m}$ ) ; mais ce sont les microphanérophytes qui dominent avec 96 espèces, soit 30,47 p.c., suivi des nanophanérophytes avec 74 espèces, soit 23,49 p.c. et des mésophanérophytes avec 24 espèces, soit 7,61 p.c. 


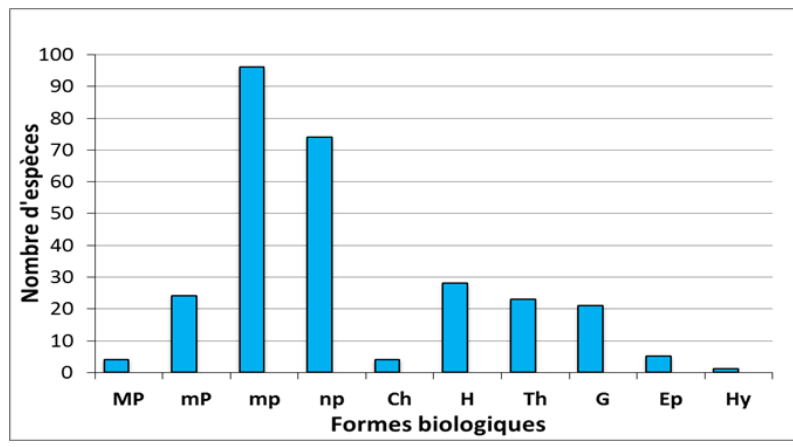

Figure 5. Spectre des types biologiques de la flore de la forêt classée de la Palé

\section{Espèces à statut particulier}

Au total 16 espèces ont été identifiées comme espèces à statut particulier dans la zone d'étude (Tableau 4). Parmi ces espèces, quatre espèces sont signalés comme endémiques des forêts de la Haute Guinée. Il s’agit de Ficus ottoniifolia, Scadoxus multiflorus, Sherbournia calycina, et Tricalysia faranahensis. Il a été comptabilisé 9 espèces rares ou menacées d'extinction selon l'UICN. Elles sont de la classe des espèces vulnérables pour 3 d'entre elles. Ce sont Afzelia africana, Khaya senegalensis et Vitellaria paradoxa. Ces espèces appartiennent à la classe des taxons dont le risque d'extinction est toujours croissant. Dans cette forêt, 3 espèces sont considérées comme rares ou menacées d'extinction de la flore ivoirienne selon les listes de Aké-Assi (1998). Ce sont : Aubrevillea platycarpa, Ensete livingstonianum et Syzygium guineense var. macrocarpum.

Tableau 4. Espèces à statut particulier rencontrées dans la forêt classée de la Palé

\begin{tabular}{|c|c|c|c|}
\hline Espèces récoltées & $\begin{array}{c}\text { Espèces } \\
\text { UICN }\end{array}$ & $\begin{array}{c}\text { Espèces Haute- } \\
\text { Guinée }\end{array}$ & $\begin{array}{c}\text { Espèces } \\
\text { Aké-Assi }\end{array}$ \\
\hline Afzelia africana Sm. & VU & & \\
\hline Aubrevillea platycarpa & & & + \\
\hline Detarium microcarpum & LC & & + \\
\hline Ensete livingstonianum & & & \\
\hline Ficus ottoniifolia & & & \\
\hline Isoberlinia doka & LC & & \\
\hline Khaya senegalensis & VU & & \\
\hline Panicum repens Linn. & LC & & \\
\hline Paspalum scobiculatum var. scrobiculatum & LC & & \\
\hline Raphia sudanica & DD & & + \\
\hline Scadoxus multiflorus & & + & \\
\hline Sherbournia calycina & & + & \\
\hline Syzygium guineense var. macrocarpum & & & \\
\hline Tacca leontopetaloides & LC & & \\
\hline Tricalysia faranahensis & & + & \\
\hline Vitellaria paradoxa & VU & & \\
\hline Total & 09 & 4 & \\
\hline
\end{tabular}




\section{Discussion}

\section{Vérification De La Classification Dirigée}

Les classifications dirigées ont permis d'obtenir des précisions cartographiques satisfaisantes pour les deux images (précisions $>90$. En effet, selon Pontius (2000), une étude d'occupation du sol peut être validée si le taux de classification global est supérieur ou égal à 50 p.c. Des résultats similaires ont été obtenus par Koné et al. (2007), dans une étude menée dans la forêt classée du Mont Korhogo situé en milieu de savane soudanienne, en Côte d'Ivoire. Cependant, des confusions, bien que mineurs, ont été enregistrées entre certaines classes d'occupation du sol. Ces confusions sont dues à des réponses spectrales similaires pour certaines formations végétales (Diallo et al., 2011 ; Tankoano et al., 2015). En effet, les savanes arborées et les savanes arbustives sont des formations mixtes qui ne sont pas toujours bien différenciées (N'guessan et al., 2003; Tankoano, 2012). De même les cultures, les friches et les savanes herbeuses sont composées d'espèces appartenant souvent aux mêmes types biomorphologiques qui peuvent présenter les mêmes signatures spectrales.

Dynamique des classes d'occupation du sol entre 2002 et 2016

L'analyse de la dynamique d'occupation du sol entre 2002 et 2016 montre que les formations boisées (forêts denses sèches/forêts galeries, forêts claires et les savanes arborées) sont en régression. Les causes de la régression des formations boisées, en Afrique de l'Ouest, sont connues. Kokou et al. (2006) ont montré qu'elles sont d'origine anthropique (feux de brousse, agriculture, surpâturage, exploitation forestière). Ces activités anthropiques, comme facteur important dans la dynamique régressive des formations végétales en zone soudanienne, ont été déjà soulignées par de nombreux auteurs comme Bamba et al. (2008), Adjonou et al. (2010), Inoussa et al. (2011) et Tankoano (2012) évoquent l'assèchement du climat ces dernières années. La forêt classée de la Palé est soumise à un climat à une seule saison pluvieuse qui est souvent marquée par des épisodes de sécheresses accentuées observées généralement en Afrique de l'Ouest (Ardoin, 2004 ; Ozer et al., 2010).

Ces périodes de déficit hydrique favorisent l'action des feux de brousse qui contribuent ainsi à dégrader la couverture ligneuse (Koné et al., 2007). Cette hypothèse parait plausible dans la mesure où on constate une augmentation des classes des affleurements granitiques et des savanes arbustives. L’occupation agricole de la forêt classée peut être appréciée par l'apparition de zones de cultures au détriment de la végétation en 2016. Cette apparition est une observation très importante car elle permet de situer la période des activités agricoles dans cette forêt mais aussi de mesurer l'impact de l'absence de l'administration forestière, due à la crise sociopolitique, au Nord de la Côte d'Ivoire, entre 2002 et 2012. 


\section{Caractérisation de la flore}

Les inventaires botaniques réalisés permettent d'établir une première ébauche de catalogue floristique de cette forêt. Les 281 espèces recensées témoignent de la richesse floristique de ce site. Cette richesse floristique est supérieure à celle obtenue par Tiébré et al. (2016) en zone soudanienne dans la localité de Korhogo (243 espèces), mais plus faible que celle obtenue par Ouattara et al. (2006), dans la zone soudanienne dans le Nord-Ouest (429 espèces), de la Côte d'Ivoire. Il s'agit de zones de savanes géomorphologiquement similaires ou plus humides que le secteur nordsoudanien. En effet, Selon Kouassi (2007), l'humidité constitue l'un des facteurs déterminants pour la diversité floristique.

Les familles botaniques dominantes sont les Fabaceae lato sensu, les Poaceae et les Rubiaceae. Cette situation est analogue à celle observée par Ouédraogo (2008) et Adjonou et al. (2009). L'explication de ces observations résiderait au niveau de la pression du pâturage qui peut modifier la composition floristique originelle la forêt classée. Les Ptéridophytes sont faiblement représentées dans la forêt classée de la Palé, avec seulement 3 espèces. Cette rareté des Ptéridophytes pourrait s’expliquer par la faible humidité de cette forêt soudanienne. En effet, selon Adou et al. (2016) et Aké Assi (2001), la forêt dense constitue le biotope favorable pour le développement de la majorité des fougères. Le nombre important de phanérophytes, obtenu dans cette étude est due, sans doute, au caractère forestier de la végétation de la forêt classée ou sans doute le résultat de leur régénération vivace par bourgeon, qui constitue un mode de régénération quantitative important dans la végétation originelle (Catinot, 1994 ; Koulibaly, 2008). L’abondance des microphanérophytes, révèle une prépondérance des formations arbustives dans la forêt classée, car les formations boisées ont connu une régression sous l'action conjuguée des Hommes et des fluctuations climatiques.

En ce qui concerne le cortège des espèces à statut particulier, les résultats montrent que ces espèces sont faiblement représentées dans la forêt étudiée. Ouattara et al. (2016) ont obtenu des résultats similaires dans la zone soudanienne du Nord-ouest de la Côte d'Ivoire. Ces auteurs attribuent cette faible représentativité de ces espèces particulières aux perturbations de la végétation, causées par les activités anthropiques ou à la faible pluviométrie de la zone (Kouassi, 2007). Cependant, ces espèces constituent des éléments importants dans la conservation de leur biodiversité (Adou Yao, 2005).

\section{Conclusion}

L’étude, réalisé dans la forêt classée de la Palé, a révélé de fortes modifications du paysage de la forêt classée de la Palé. Les formations boisées ont connu une régression, entre 2002 et 2016, entrainant ainsi une 
modification profonde dans la structure spatiale de la forêt. Les causes majeures de cette perte des formations boisées peuvent être les défrichements agricoles, les feux de brousse et les activités pastorales.

Concernant l'étude floristique, elle a permis de mettre en évidence une richesse spécifique intéressante (281 espèces). Les résultats montrent que les inventaires restent le seul outil efficace pour l'évaluation et la valorisation des ressources naturelles. Les 18 espèces à statut particulier identifiées témoignent de la valeur pour la conservation de la forêt classée.

Ces résultats constituent une première étape dans l'établissement d'une base de données sur la flore et la végétation des forêts classées de la Région de la Bagoué. Son approfondissement et son extension permettront de réactualiser la carte bioclimatique et d'amorcer la rédaction de la flore des forêts classées du Nord de la Côte d’Ivoire.

\section{References:}

1. Adjonou, K., Bellefontaine, R., \& Kokou, K. (2009). Les forêts claires du Parc National Oti-Kéran au Nord-Togo: structure, dynamique et impacts des modifications climatiques récentes. Sécheresse, 20(1) : 110. DOI : 10.1684/sec.2009.0211

2. Adou Yao, C. Y. (2005). Pratiques paysannes et dynamiques de la biodiversité dans la forêt classée de Monogaga (Côte d'Ivoire). Thèse de Doctorat unique, Département Hommes Natures et Société, Université MNHN, Paris, France, 233 p.

3. Adou, D. M. L., Ipou, I. J., \& Adou Yao, Y. C. (2016). Répartition écologique des Ptéridophytes dans le Sud-Est de la Côte d'Ivoire. International Journal of Innovation and Applied Studies, 17(1) : 320330. DOI : 10.4314/ijbcs.v4i1.54242

4. Aké Assi, L. (1984). La flore de la Côte d'Ivoire : étude descriptive et biogéographique, avec quelques notes ethnobotaniques. Tome I, II, III. Thèse de Doctorat d'Etat ès Sciences, Université Nationale de Côte d'ivoire, $1205 \mathrm{p}$.

5. Aké Assi, L. (2001). Flore de la Côte d'Ivoire 1, catalogue, systématique, biogéographie et écologie. Conservatoire et Jard. Bot., Genève, Switzerland, Boissiera, 57 : 1-396.

6. Aké Assi, L. (2002). Flore de la Côte d'Ivoire 2, catalogue, systématique, biogéographie et écologie.

7. Ardoin, B. S. (2004). Variabilité hydroclimatique et impacts sur les ressources en eau de grands bassins hydrographiques en zone soudanosahélienne. Thèse de l’Université de Montpellier II, France, 330 p.

8. Balac, R. (2000). Les économies pionnières prédatrices du milieu forestier : le cas de l'économie de plantation en Côte d'Ivoire. Travaux de la Société d'Écologie Humaine, pp. 429-438. 
9. Bamba, I., Mama, A., Neuba, D. F. R., Koffi, K. J., Traoré, D., Visser, M., Sissin, B., Lejoly, J. \& Bogaert, J. (2008). Influence des actions anthropiques sur la dynamique spatiotemporelle de l'occupation du sol dans la province du Bas-Congo (R.D. Congo). Sciences et Nature, 5(1) : 49-60. DOI : $10.4314 /$ scinat.v5i1.42151

10. Bonn, F. \& Rochon, G. (1993). Précis de télédétection : principes et méthodes. Presses de l'Université du Québec. Sainte-Foy., Canada, $485 \mathrm{p}$.

11. Catinot, R. (1994). Aménager les savanes boisées africaines. Un tel objectif semble désormais à notre portée. Bois et Forêts des Tropiques, 241 : 53-67. DOI : https://doi.org/10.19182/bft1994.241.a19812

12. Diallo, H., Barnba, I., Barirna, Y. S. S., Visser, M., Ballo, A., Marna, A., Vranken, I., Maïga, M. \& Bogaert, J. (2011). Effets combinés du climat et des pressions anthropiques sur la dynamique évolutive de la végétation d'une zone protégée du Mali (Réserve de Fina, Boucle du baoulé). Sécheresse, 22(3) : 97-107.

DOI : http://hdl.handle.net/2268/106573

13. Enonzan, F. B. (2010). Utilisation de la télédétection et des SIG dans la gestion durable des aires protégées : cas des forêts classées de DogoKetou au Bénin. Mémoire de fin d'études, Obafemi Awolowo University Campus, Nigéria. 89 p.

14. Grégroire, J. M. \& Simonetti, D. (2010). Interannual changes of fire activity in the Protected area of the SUN (Sustainable use of natural vegetation in West Africa) Network and other Parks and Reserves of the West and Central Africa Region derived from MODIS observations. Ecological Status and Change by Remote Sensing, 2(2): 446-463. DOI : https://doi.org/10.3390/rs2020446

15. Hahn-Hadjali, K. (1998). Les groupements végétaux des savanes du Sud- Est du Burkina Faso (l'Afrique de l'Ouest). - Études sur la flore et la végétation du Burkina Faso et des pays avoisinants, $3: 3-79$.

16. Hien, M., Boussim, J., \& Guinko, S. (2002). L'utilisation de Burkea africana Hook. (Caesalpiniaceae) par les populations d'éléphants Loxodonta africana (Blumen) Bach. dans le Ranch de Gibier de Nazinga (Burkina Faso). Annales de Botanique de l'Afrique de l'Ouest, 2 : 25-35.

17. Holmgren, M., Poorter, L., Siegel, A., Bongers, F., Buitelaar, M., Chatelain, C., Gauthier, L., Hawthorne, W. D., Helmink, A. T. F., Jongkind, C. C. H., Os-Breijer, H. J., Weirenga, J. J. \& Van Zoest, A. R. (2004). Ecological profiles of rare and endemic species. In : Biodiversity of West African forests, an Ecological Atlas of Woody plant Species, CABI Publiching, Cambridge, pp. 101-389. 
18. Inoussa, M. M., Mahamane, A., Mbow, C., Saadou, M. \& Yvonne, B. (2011). Dynamique spatiotemporelle des forêts claires dans le Parc national du W du Niger (Afrique de l'Ouest). Sécheresse, 22(2) : 108116.

DOI :http://pascalfrancis.inist.fr/vibad/index.php?action=getRecordD etail\&idt $=24247762$

19. Kokou, K., Atato, A., Bellefontaine, R., Kokutse, A. D. \& Caballé, G. (2006). Diversité des forêts denses sèches du Togo. Revue d'Ecologie, 61(3) : 225-246. DOI : http://hdl.handle.net/2042/55682

20. Koné, M., Aman, A., Yao, A. C., Coulibaly, L. \& N’Guessan, K. E. (2007). Suivi diachronique par télédétection spatiale de la couverture ligneuse en milieu de savane soudanienne en Côte d'Ivoire. Revue Télédétection, 7(1-2-3-4) : 433-446.

21. Kouamé, N. F. (1998). Influence de l'exploitation forestière sur la végétation et la flore de la forêt classée du Haut-Sassandra (CentreOuest de la Côte d'Ivoire). Thèse de Doctorat $3^{\text {ème }}$ Cycle, UFR Biosciences, Université Cocody- Abidjan, Côte d'Ivoire, 227 p.

22. Kouassi, K. E. (2007). Flore de la forêt classée de la Haut-Dodo, dans le Sud-ouest de la Côte d'Ivoire. Etude de quelques espèces commerciales : cas de Garcinia afzelii (Clusiaceae), des rotins (palmiers lianes) des genres Calamus, Eremospatha et Laccosperma (Arecaceae). Thèse de Doctorat., UFR Biosciences, Université de Cocody-Abidjan, Côte d'ivoire, 279 p.

23. Koulibaly, A.V. (2008). Caractéristique de la végétation et dynamique de la régénération, sous l'influence de l'utilisation des terres, dans des mosaïques forêts-savanes, des Régions de la Réserve de Lamto et du Parc National de la Comoé, en Côte d'Ivoire. Thèse de Doctorat, UFR Biosciences, Université de Cocody-Abidjan, Côte d'Ivoire, 137 p.

24. Léonard, E. \& Ibo, J. G. (1994). Appropriation et gestion de la rente forestière en Côte d'Ivoire. Politique Africaine 53 : 25-36.

25. Lykke, A.M. (2000). Local perception of vegetation change and priorities for conservation of woody savanna vegetation in Senegal. Journal of Environmental Management, 59: 107-120.

26. N'guessan, E., Behan, M. F. \& Blasco, F. (2003). Suivi par télédétection spatiale d'une forêt tropicale humide protégée soumise à des pressions anthropiques. Télédétection, 3(5) : 443-456.

27. Ouattara, D., Kouamé, D., Tiebré, M. S., Cissé, A. \& N’Guessan, K. E. (2016). Diversité floristique et usages des plantes dans la zone soudanienne du Nord-ouest de la Côte d'Ivoire. Journal of Animal \& Plant Sciences, 31 : 4815-4830.

DOI : https://www.academia.edu/42807156/ 
28. Ouédraogo, M. (2008). Les ripicoles forestières de la Réserve de la Biosphère de la Mare aux Hippopotames du Burkina Faso : caractéristiques, dynamique et ethnobotanique. Thèse de Doctorat de l’Université de Ouagadougou, Burkina Faso, 262 p.

29. Ouôba, P., Boussim, J. \& Guinko, S. (2006). Le potentiel fruitier de la forêt classée de Niangoloko au Burkina Faso. Fruits, 61 : 71-81.

30. Ozer, P., Hountondji, Y. C., Niang, A. J., Karimoune, S., Manzo, O. L. \& Salmon, M. (2010). Désertification au Sahel : historique et perspectives. Bulletin de la Société Géographie de Liège, 54 : 69-84. DOI : https://popups.uliege.be:443/0770-7576/index.php?id=942.

31. Pontius Jr., R. G. (2000). Quantification error versus location error in comparison of categorical maps. Photogrammetric Engineering and remote Sensing, 66(8) : 1011-1016.

DOI : http://worldcat.org/issn/00991112

32. Tankoano, B. (2012). Suivi diachronique de la couverture ligneuse dans la forêt classée de Koulbi à l'aide de la télédétection et des systèmes d'information géographique (SIG). Mémoire IDR, Université Polytechnique de Bobo-Dioulasso, Burkina Faso, 94 p.

33. Tankoano, B., Hien, M., Dibi, N. H., Sanon, Z., Yameogo, J. T. \& Somda, I. (2015). Dynamique spatio-temporelle des savanes boisées de la forêt classée de Tiogo au Burkina Faso International Journal of Biological and Chemical Sciences, 9(4) : 1983-2000. DOI : 10.4314/ijbcs.v9i4.23

34. Tente, B., \& Sinsin, B. (2002). Diversité et structure des formations arborescentes du secteur Perma-Toucountouna dans la chaîne de l'Atacora (Bénin). Études sur la Flore et la Végétation du Burkina Faso, $6: 31-42$.

35. Thiombiano, A. (1996). Contribution à l'étude des Combretaceae dans les formations végétales de la région Est du Burkina Faso. Thèse de Doctorat $3^{\text {ème }}$ cycle, Université de Ouagadougou, Burkina Faso, 220 p.

36. Tiébré, M. S., Ouattara, D., Vroh, B. T. A., Gnagbo, A. \& N'Guessan, K. E. (2016). Diversité floristique et disponibilité des plantes utilitaires en zone soudanienne de la Côte d'Ivoire. Journal of Applied Biosciences, 102 : 9699-9707. DOI : 10.4314/jab.v102i1.4

37. UICN (2015). UICN Red List of Threatened Species. Version 2015.1. $<$ www.iucnredlist.org>. 